\title{
Stability at high temperature and decomposition kinetics of the fullerene dimers and photopolymers
}

\author{
K. P. Meletov ${ }^{1 *}$, J. Arvanitidis ${ }^{2}$, D. Christofilos ${ }^{2}$, G. Kourouklis² ${ }^{2}$, V. A. Davydov ${ }^{3}$ \\ ${ }^{1}$ Institute of Solid State Physics, Russian Academy of Sciences, 142432 Chernogolovka, Moscow region, Russia \\ ${ }^{2}$ Aristotle University of Thessaloniki, GR-54124 Thessaloniki, Greece \\ ${ }^{3}$ Institute of High Pressure Physics, Russian Academy of Sciences, 42092 Troitsk, Moscow region, Russia \\ *mele@issp.ac.ru
}

PACS 78.30.Na

DOI 10.17586/2220-8054-2018-9-1-29-32

\begin{abstract}
The decomposition kinetics of the fullerene dimers and photo-oligomers was studied at elevated temperature by Raman scattering. The polymeric content decreases exponentially with the thermal treatment time while the decay time constant decreases at higher temperatures. The activation-type behavior is well described by the Arrhenius law that gives the activation energy $E_{A}=(1.71 \pm 0.06) \mathrm{eV} / \mathrm{molecule}$ for the dimers and $E_{A}=(0.87 \pm 0.06) \mathrm{eV} /$ molecule for the $\mathrm{C}_{60}$ photopolymer.
\end{abstract}

Keywords: fullerene polymers, Raman spectroscopy, decomposition kinetics.

Received: 14 June 2017

Revised: 22 September 2017

\section{Introduction}

The photopolymerization of $\mathrm{C}_{60}$ occurs in thin films or surfaces of bulk samples resulting in rather disordered oligomers $\left(\mathrm{C}_{60}\right)_{n}$ that appear via the [2+2] cyclo-addition mechanism [1-3]. The treatment of $\mathrm{C}_{60}$ under various conditions of high pressure and high temperature (HPHT) results in the formation of bulk polymers $[4,5]$. The lowering of the $\mathrm{C}_{60}$ molecular symmetry leads to the splitting and softening of phonon bands and the Raman examination of polymers is based on the behavior of the $A_{g}(2)$ pentagon pinch (PP) mode frequency which decreases from $1468 \mathrm{~cm}^{-1}$ for the $\mathrm{C}_{60}$ monomer to $1463,1458,1446$ and $1408 \mathrm{~cm}^{-1}$ for the dimers, linear orthorhombic, planar tetragonal and rhombohedral polymers, respectively $[5,6]$. Fullerene polymers are stable at ambient conditions but revert to the monomeric state at elevated temperature $[7,8]$. The differential scanning calorimetry (DSC) of the HPHT polymers shows transitions in the range $170-280{ }^{\circ} \mathrm{C}$ which depend on the polymeric phase and somewhat on the scanning rate, indicating that the process is controlled by kinetics. The decomposition kinetics of polymers was studied by X-ray thermal expansion and Raman measurements [7,9]. In the case of photopolymers, the small amount of the photo- transformed material on the surfaces of samples makes the DSC and X-ray measurements inappropriate, so the Raman spectroscopy becomes a unique tool in the study of the photopolymer decomposition. Here, we report the Raman study of the decomposition kinetics of the $\mathrm{C}_{60}$ dimers and photopolymers. The intensity of the polymer-related PP-mode decreases exponentially with the thermal treatment time. Decomposition becomes faster at elevated temperatures and the activation energy $E_{A}$, obtained from the Arrhenius dependence of the exponential decay time constant on temperature, is $(1.71 \pm 0.06)$ and $(0.87 \pm 0.12) \mathrm{eV}$ for dimers and photopolymers, respectively.

\section{Experimental details}

The dimeric samples, prepared by HPHT treatment of pristine $\mathrm{C}_{60}$, were a mixture $4: 1$ of $\mathrm{C}_{60}$ dimer and monomer [10]. The $\mathrm{C}_{60}$ photopolymer was formed under laser irradiation at the wavelength $\lambda_{e x c}=785 \mathrm{~nm}$ and $180 \mu \mathrm{W}$ power. The photopolymer decomposition was studied by Raman probing at $\lambda_{e x c}=785 \mathrm{~nm}$ with $18 \mu \mathrm{W}$ power under continuous thermal treatment up to $400 \mathrm{~min}$ at an elevated temperature. The Raman measurements were performed in the back-scattering geometry using a LabRam HR spectrometer and a high temperature cell with a quartz window equipped by a temperature controller unit [11]. 


\section{Results and discussion}

The dimeric samples are sensitive to visible light and may be subjected to further photopolymerization during long acquisition Raman experiments. On the other hand, the spectra recorded at $\lambda_{e x c}=785 \mathrm{~nm}$ and laser power of $80 \mu \mathrm{W}$ do not show any changes for irradiation time up to $200 \mathrm{~min}$. The kinetics of dimer decomposition was studied at these conditions under thermal treatment at temperatures ranging from $130-177{ }^{\circ} \mathrm{C}$. The Raman spectra were measured in the frequency region of the PP-mode with fixed acquisition time, consecutively, during continuous thermal treatment, using a new fresh sample for each temperature.

The inset of Fig. 1 depicts the evolution of the Raman spectra of the $\mathrm{C}_{60}$ dimers upon thermal treatment at $155^{\circ} \mathrm{C}$. In the bottom spectrum, recorded at ambient conditions, the PP-mode component of dimers is dominant, while subsequent spectra recorded at $155{ }^{\circ} \mathrm{C}$ exhibit an intensity increase of the monomer component at the expense of the dimer one. The top spectrum recorded after $97 \mathrm{~min}$ of thermal treatment is dominated by the PP-mode component of the monomer while the dimer component becomes negligible. Open circles in Fig. 1 represent the fractional intensity of the dimer PP-mode as a function of the thermal treatment time, whereas the dotted line is the fit of the data by the exponential decay function:

$$
D(t)=D_{0} \exp (-t / \tau)
$$

where $D(t)$ and $D_{0}$ are the current and the initial contents of dimers and $\tau$ is the decay time constant.

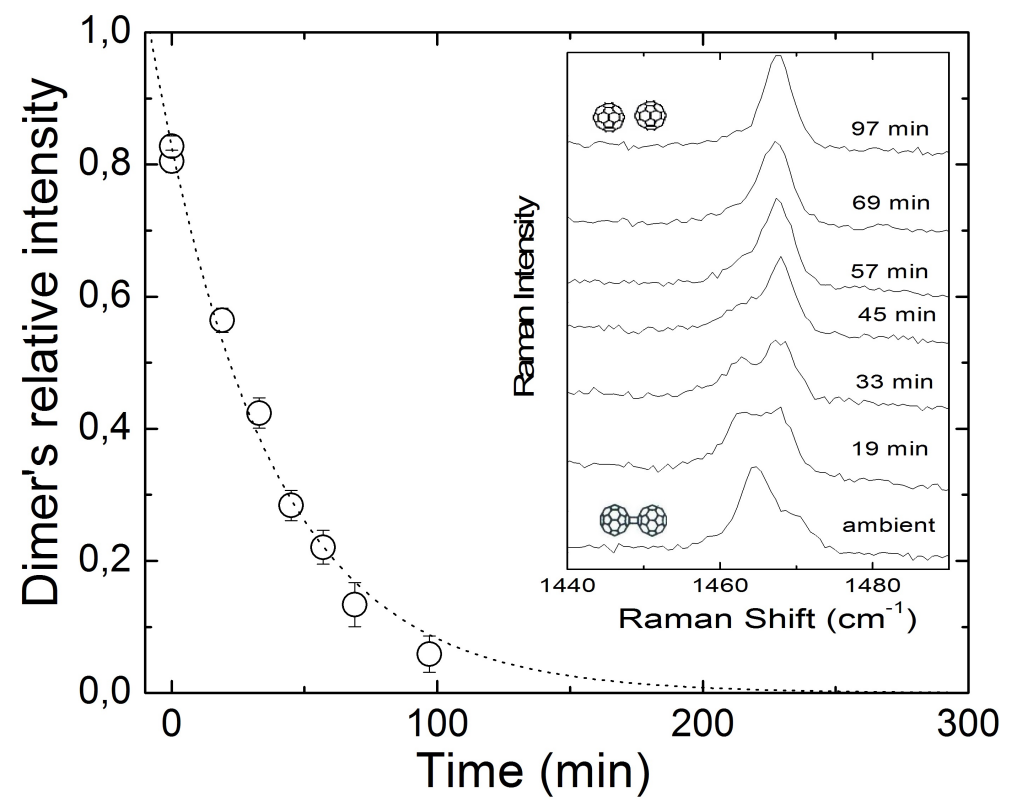

FIG. 1. Evolution of the Raman spectra of the $\mathrm{C}_{60}$ dimers under thermal treatment at $155{ }^{\circ} \mathrm{C}$

Figure 2 illustrates the decomposition kinetics of dimers, which is relatively slow at $135{ }^{\circ} \mathrm{C}$ with $\tau=419$ min, while it becomes very fast at the highest temperature of $177{ }^{\circ} \mathrm{C}$ with $\tau=6.9 \mathrm{~min}$. The activation energy of the dimer decomposition reaction can be described by the Arrhenius equation:

$$
\tau(T)=A \exp \left(E_{A} / k_{B} T\right),
$$

where $E_{A}$ is the energy barrier between the dimeric and the monomeric states, $k_{B}$ is the Boltzmann constant, $T$ is the treatment temperature and $\tau$ the decay time constant. The constant $A$, related to the characteristic phonon frequency, is measured in time units [9]. The corresponding Arrhenius plot is included in the Fig. 2 inset. The experimental data exhibit a good linear dependence in logarithmic scale, yielding an activation energy of $E_{A}=(1.71 \pm 0.06) \mathrm{eV} /$ molecule. This value is close to the $E_{A}=(1.75 \pm 0.05) \mathrm{eV} / \mathrm{molecule}$ obtained from the X-ray thermal expansion studies of powdered dimeric samples, synthesized by a solid-state mechanochemical reaction of $\mathrm{C}_{60}$ with potassium cyanide, as well as to the activation energy $E_{A}=(1.9 \pm 0.1) \mathrm{eV} / \mathrm{molecule}$ of the $1 \mathrm{D}$ and 2D polymers decomposition [9]. It is interesting to compare these data with the decomposition kinetics of fullerene photooligomers $\left(\mathrm{C}_{60}\right)_{n}$. The Raman spectra of the photopolymerized fullerene prepared at $\lambda_{\text {exc }}=532 \mathrm{~nm}$ and $60 \mu \mathrm{W}$ laser power are shown in the left inset of Fig. 3. The initial spectrum recorded in the region of the $A_{g}(2)$ PP-mode is typical for the $\mathrm{C}_{60}$ monomer while the following spectra recorded with 60 min exposure under 


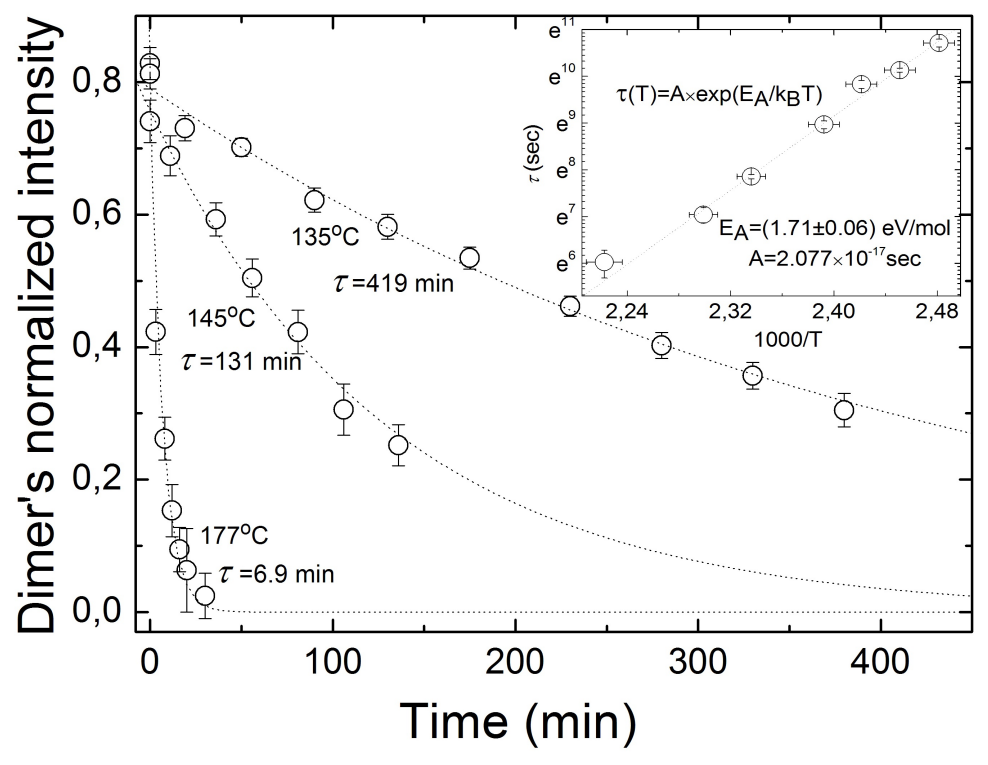

FIG. 2. Decomposition kinetics of the $\mathrm{C}_{60}$ dimers

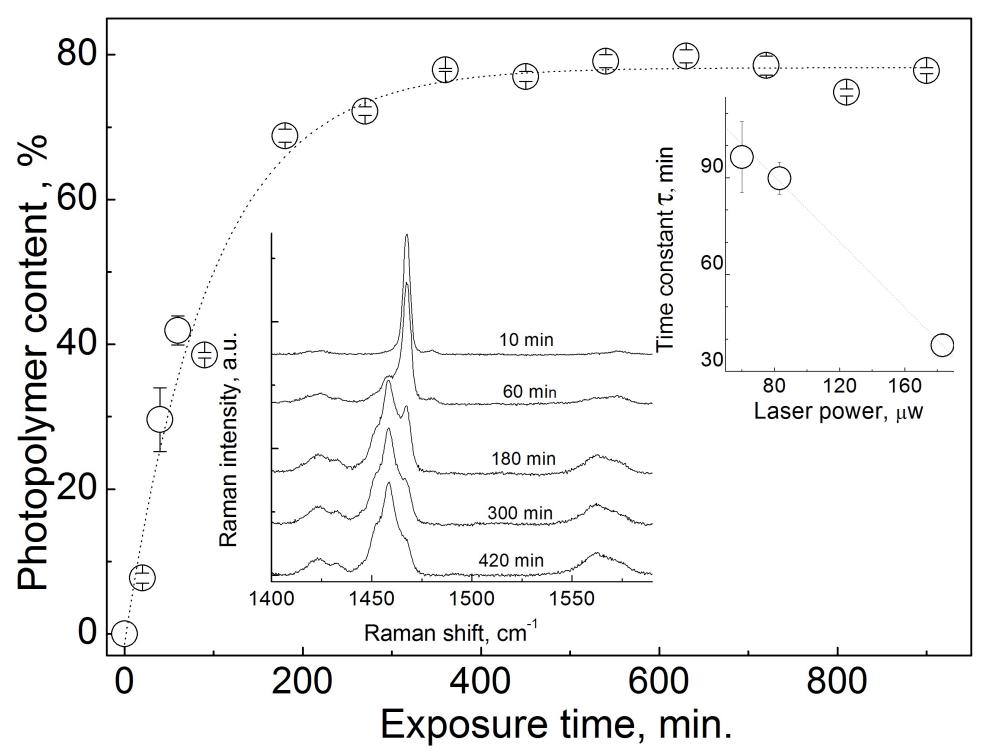

FIG. 3. Raman spectra and photopolymerization kinetics of the $\mathrm{C}_{60}$ fullerite

continuous illumination show gradual decrease of the PP-mode intensity of the monomer and increase of the new PP-modes intensity. The intensity distribution between the new modes shows that photo-oligomers are mainly linear chains and conjugated linear chains. Open circles depict the dependence of the photopolymer content on the irradiation time whereas the dotted line is the fit by the exponential growth function:

$$
P(t)=P_{\text {sat }}\{1-\exp (-t / \tau)\}
$$

where $P(t)$ and $P_{\text {sat }}$ are the current and the saturated contents of the photopolymer, $\tau$ is the time constant that linearly decreases with laser power (right inset).

The stability of the photo-oligomers, prepared on the surface of the $\mathrm{C}_{60}$ crystals at $\lambda_{\text {exc }}=785 \mathrm{~nm}$ and laser power $180 \mu \mathrm{W}$, was studied by Raman spectroscopy with $\lambda_{e x c}=785 \mathrm{~nm}, 18 \mu \mathrm{W}$ and short acquisition time to prevent further polymerization and ensure accurate measurements.

Figure 4 illustrates the kinetics of the photo-oligomer decomposition. The relatively slow decomposition at $130{ }^{\circ} \mathrm{C}$ with $\tau=109 \mathrm{~min}$ becomes very fast at $170{ }^{\circ} \mathrm{C}$ with $\tau=9.6 \mathrm{~min}$. The activation energy of the decomposition can be estimated from the Arrhenius plot included in the Fig. 4 inset. The experimental data exhibit a good 
linear dependence in logarithmic scale, yielding an activation energy of $E_{A}=0.87 \pm 0.06 \mathrm{eV} / \mathrm{molecule}$. Note that a value of $1.25 \mathrm{eV} /$ molecule was obtained earlier in the Raman study of the $\mathrm{C}_{60}$ photopolymer decomposition [7]. The Arrhenius data allows the estimation of the photopolymer complete decomposition temperature (decrease of its content to $1 \%$ within $15 \mathrm{~min}$ as in DSC experiments [8]). Despite of the twice as large activation energy of the $\mathrm{C}_{60}$ dimers, their complete decomposition temperature is $\sim 180{ }^{\circ} \mathrm{C}$, lying close to that of $\sim 190{ }^{\circ} \mathrm{C}$ for the photopolymers due to the giant difference in the $A$ constant of the Arrhenius dependence. Finally, the recent study of the photopolymerization in the fullerene layers of the $\left\{\mathrm{Pt}(\mathrm{dbdtc})_{2}\right\} \cdot \mathrm{C}_{60}$ complex shows that the activation energy of the layered photopolymer decomposition is $E_{A}=1.12 \pm 0.11 \mathrm{eV} /$ molecule while the temperature of its complete decomposition is $\sim 130{ }^{\circ} \mathrm{C}$ [12]. Note, that the $2 \mathrm{D}$ polymers appear to be the most stable among the polymeric forms of $\mathrm{C}_{60}$, characterized by a complete decomposition temperature of $\sim 260{ }^{\circ} \mathrm{C}$ [8].

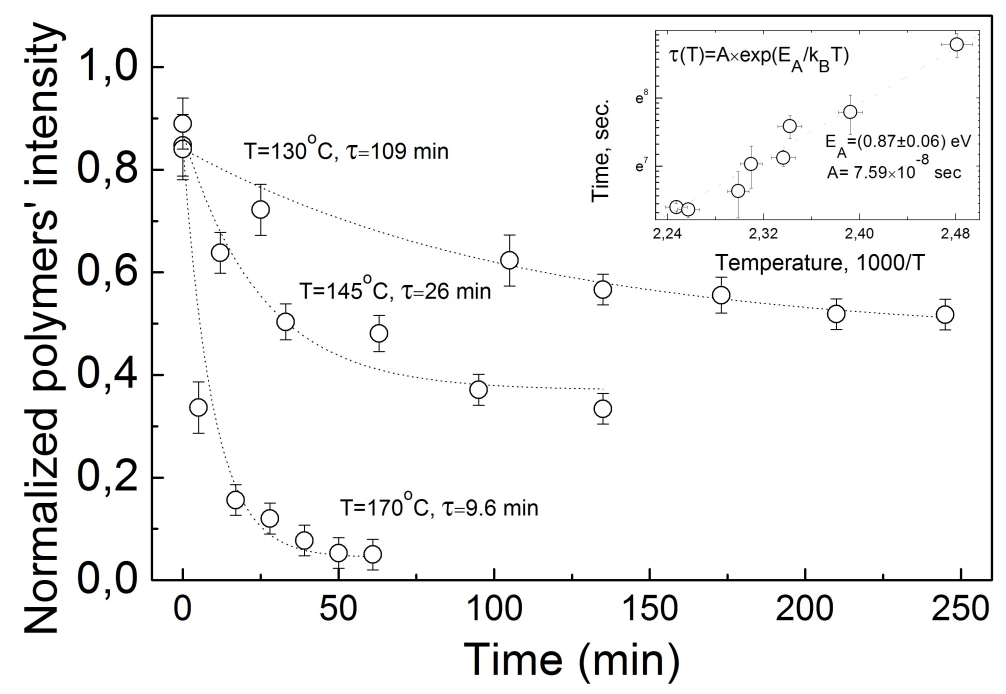

FIG. 4. Time dependencies of the $\mathrm{C}_{60}$ photopolymer content under treatment at various temperatures and their fit by exponential decay functions (dotted lines). Inset: Arrhenius dependence of the decay time constant on temperature

\section{Acknowledgements}

The authors are thankful to the Center of Interdisciplinary Research and Innovation of the Aristotle University of Thessaloniki (KE $\Delta \mathrm{EK}$ ) for the access to the Raman instrumentation. K.P.M. acknowledges the main financial support by the Federal Agency for Scientific Organizations, the partial support by the Russian Foundation for Fundamental Research, grant 15-02-01495, the RAS Presidium Program "Physics of compressed matter", as well as the hospitality of the Aristotle University of Thessaloniki.

\section{References}

[1] Rao A.M., Zhou P., et al. Photoinduced polymerization of solid C60 films. Science, 1993, 259, P. 955-957.

[2] Zhou P., Dong Z.H., Rao A.M., Ecklund P.C. Reaction-mechanism for photopolymerization of solid fullerene C 60 . Chem. Phys. Lett., 1993, 211, P. 337-340.

[3] Pusztai T., Oszlanyi G., et al. Bulk structure of phototransformed C60. Solid State Commun., 1999, 111, P. 595-599.

[4] Nunez-Regueiro M., Marques L., et al. Polymerized fullerite structures. Phys. Rev. Lett., 1995, 74, P. $278-281$.

[5] Iwasa Y., Arima T., et al. New phases of $\mathrm{C}_{60}$ synthesized at high pressure. Science, 1994, 264, P. 1570-1572.

[6] Senyavin V.M., Davydov V.A., et al. Spectroscopic properties of individual pressure-polymerized phases of C 60. Chem. Phys. Lett., 1999, 313, P. 421-425.

[7] Wang Ying, Holden J.M., Bi Xiang-xin, Ecklund P.C. Thermal decomposition of polymeric C 60. Chem Phys. Lett., 1994,217, P. $413-417$.

[8] Iwasa Y., Tanoue K., Mitani T., Yagi T. Energetics of polymerized fullerites. Phys. Rev. B, 1998, 58, P. 16374-16377.

[9] Nagel P., Pasler V., et al. $\mathrm{C}_{60}$ one- and two-dimensional polymers, dimers, and hard fullerite: Thermal expansion, anharmonicity, and kinetics of depolymerization. Phys. Rev. B, 1999, 60, P. 16920-16927.

[10] Moret R., Launois P., et al. Single-crystal study of the pressure-temperature-induced dimerization of C60. Eur. Phys. J. B, 2004, 37, P. 25-37.

[11] Meletov K.P., Krestinin A.V., et al. Temperature effects in the Raman spectra of bundled single-wall carbon nanotubes. Chem. Phys. Lett., 2009, 477, P. 336-339.

[12] Meletov K.P., Velkos G.,et al. Raman study of the photopolymer formation in the $\left.\{\text { Pt(dbdtc })_{2}\right\} \cdot \mathrm{C}_{60}$ fullerene complex and the decomposition kinetics of the photo-oligomers. Chem. Phys. Lett., 2017, 681 (1), P. 124-129. 